\title{
Melatonin prevents hyperglycemia in a model of sleep apnea
}

Graduate Program in Medical Sciences, Universidade Federal do Rio Grande do Sul (UFRGS), Porto Alegre, RS, Brazil ${ }^{2}$ Cardiology Division, Hospital de Clinicas de Porto Alegre (HCPA), UFRGS, Porto Alegre, RS, Brazil ${ }^{3}$ Graduate Program in Cardiology and Cardiovascular Sciences, UFRGS, Porto Alegre, RS, Brazi

${ }^{4}$ Faculdade Cenecista de Bento Gonçalves, Bento Gonçalves, RS, Brazil Universidade Luterana do Brasil, Canoas, RS, Brazil

Study conducted at the Animal Experimentation Unit, HCPA, UFRGS, Brazil

\author{
Correspondence to: \\ Micheli Fagundes \\ Rua Ramiro Barcelos, 2350 \\ 90035-903 - Porto Alegre, RS, Brazil \\ michelifagundes87@gmail.com \\ Received on June/17/2014 \\ Accepted on Dec/18/2014 \\ DOI. 10.1590/2359-3997000000012
}

\author{
Renata Schenkel Rivera Kaminski', Denis Martinez ${ }^{1,2,3}$, \\ Micheli Fagundes ${ }^{3}$, Emerson Ferreira Martins ${ }^{3}$, Carolina Caruccio Montanari', \\ Darlan Pase Rosa ${ }^{1,4}$, Cintia Zappe Fiori ${ }^{2,3}$, Norma Possa Marroni ${ }^{5}$
}

\begin{abstract}
Objective: Obstructive sleep apnea is a common disorder associated with aging and obesity. Apneas cause repeated arousals, intermittent hypoxia, and oxidative stress. Changes in glucolipidic profile occur in apnea patients, independently of obesity. Animal models of sleep apnea induce hyperglycemia. This study aims to evaluate the effect of the antioxidants melatonin and $\mathrm{N}$-acetylcysteine on glucose, triglyceride, and cholesterol levels in animals exposed to intermittent hypoxia. Materials and methods: Two groups of Balb/c mice were exposed to intermittent hypoxia $(n=36)$ or sham intermittent hypoxia $(n=36)$ for 35 days. The intermittent hypoxia group underwent a total of 480 cycles of 30 seconds reducing the inspired oxygen fraction from $21 \%$ to $7 \pm 1 \%$ followed by 30 seconds of normoxia, during 8 hours daily. Melatonin or $\mathrm{N}$-acetylcysteine were injected intraperitonially daily from day 21 on. Results: At day 35, glucose levels were significantly higher in the intermittent hypoxia group than in the control group. The intermittent hypoxia groups receiving $\mathrm{N}$-acetylcysteine and vehicle showed higher glucose levels than the group receiving melatonin. The lipid profile was not affected by intermittent hypoxia or antioxidant administration. Conclusions: The present results suggest that melatonin prevents the well-recognized increase in glucose levels that usually follows exposure to intermittent hypoxia. Further exploration of the role of melatonin in sleep apnea is warranted. Arch Endocrinol Metab. 2015;59(1):66-70
\end{abstract}

Keywords

Melatonin; N-acetylcysteine; glucose; triglycerides; intermittent hypoxia

\section{INTRODUCTION}

$\mathrm{O}$ bstructive sleep apnea causes repeated arousals and intermittent hypoxia. Such events lead to sympathetic hyperactivity, oxidative stress, and insulin resistance (1). Investigation of obstructive sleep apnea (OSA) has been recommended as part of the routine care of individuals with diabetes $(2,3)$ because OSA impairs diabetes control, independently of confounding factors such as obesity (4).

The repeated hypoxic episodes seen in OSA increase oxidative stress (5), which may represent a link between apneas and diabetes (6-8). Intermittent hypoxia, a model of OSA, induces acute insulin resistance in healthy humans (9) and animals (10). Mice receiving obesity-inducing diets showed a greater increase of the HOMA index under intermittent hypoxia than lean mice (11).

In hamsters subjected to four weeks of intermittent hypoxia, melatonin prevented vascular damage resulting from the procedure (12). Melatonin-mediated protection against impairment or damage caused by intermittent hypoxia in diabetes may be related to the regulation of reactive oxygen species (13) and/or to influences of melatonin on insulin secretion (14). The antioxidant $\mathrm{N}$-acetylcysteine, a precursor of $\mathrm{L}$-cysteine, is a scavenger of reactive oxygen species (15) with a potential to influence on certain aspects of the diabetes mechanisms $(16,17)$.

To test the hypothesis that antioxidants have a role in preventing the derangements of glucolipidic profile caused by intermittent hypoxia, we conducted an experimental comparative study that evaluated the effect of the antioxidants melatonin and $\mathrm{N}$-acetylcysteine on glucose, triglyceride, and cholesterol levels in mice subjected to intermittent hypoxia during 35 days.

\section{MATERIALS AND METHODS}

\section{Animals}

At the institutional animal experimentation unit, two month-old Balb/C male mice were fed commercial standard chow (Purina-Nutripal, Brazil) and water 
ad libitum under the supervision of a veterinarian. Mice were kept six each in cages measuring $30 \times 19$ $\mathrm{x} 13 \mathrm{~cm}$, at an average room temperature of $22.4^{\circ} \pm$ $0.6^{\circ} \mathrm{C}$ and controlled humidity, on a standard $12 / 12$ hour light-dark cycle (lights on at 0700 , lights out at 1900). This method was described previously $(18,19)$. All procedures were performed in accordance with the Guide for the Care and Use of Laboratory Animals (20) and were approved by the institutional animal care committee, Hospital de Clínicas de Porto Alegre (CEUA/HCPA \#2009/09-300).

\section{Intermittent hypoxia system}

The intermittent hypoxia procedure has been described previously in adult male mice $(18,19)$, a mixture of $90 \% \mathrm{~N}_{2}$ and $10 \% \mathrm{CO}_{2}$ was released into the cages over a period of 30 seconds, gradually reducing the oxygen fraction to $7 \pm 1 \%$ and increasing the $\mathrm{CO}_{2}$ fraction to approximately $5 \pm 1 \%$; fans were then operated for 30 seconds so as to introduce room air into the cage. Overall, 480 such cycles of hypoxia-normoxia occurred per day. Thirty-six mice were allocated to the hypoxia procedure. The 36 control animals underwent the same manipulation as the hypoxia animals, but room air was insufflated into the cages instead of the aforementioned gas mixture. The groups were housed under the hypoxia system 8 hours per day (lights on at 0900AM, lights out at $0500 \mathrm{PM}$ ).

\section{Antioxidant administration}

Once daily from day 21 to day 35 , before 7:00 PM, all animals received intraperitonially injections of either 1 ) vehicle $(\mathrm{n}=24), 2)$ melatonin $(\mathrm{n}=24)$, or 3$) \mathrm{N}$-acetylcysteine $(\mathrm{n}=24)$. Melatonin was administered at a dose of $0.2 \mathrm{mg} / \mathrm{kg}$ diluted in $0.4 \mathrm{~mL}$ of $0.9 \%$ saline plus 0.1 $\mathrm{mL}$ of $1 \%$ ethanol (21). $\mathrm{N}$-acetylcysteine was administered at a dose of $10 \mathrm{mg} / \mathrm{kg}$ dissolved in $0.5 \mathrm{~mL} 0.9 \%$ saline.

\section{Protocols}

Groups of 12 mice were randomly allocated to six different protocols: 1) sham hypoxia plus vehicle injection; 2) sham hypoxia plus melatonin injection; 3) sham hypoxia plus $\mathrm{N}$-acetylcysteine injection; 4) intermittent hypoxia plus vehicle injection (hypoxia plus vehicle); 5 ) intermittent hypoxia plus melatonin injection (hypoxia plus melatonin); or 6) intermittent hypoxia plus $\mathrm{N}$-acetylcysteine injection (hypoxia plus $\mathrm{N}$-acetylcysteine). The protocols were designed to obtain better data analysis, having greater control over the experiment. All animals were weighed at the baseline, at the day 21 and 35 days $(18,19)$ using a digital scale with an accuracy of $0.01 \mathrm{~g}$ (Marte, model AS 5500C).

At day 35, after fasting for at least 8 hours, mice were anesthetized with ketamine $(100 \mathrm{mg} / \mathrm{kg})$ and xylazine $(50 \mathrm{mg} / \mathrm{kg})$. After anesthesia was confirmed, animals were euthanized (22) by the withdrawal of approximately $1 \mathrm{~mL}$ of blood for biochemical tests.

\section{Biochemical analyses}

Plasma glucose, triglyceride, and cholesterol levels were determined by enzymatic colorimetric assays using commercial kits according to manufacturer instructions. (Roche Diagnostics, Mannheim, Germany: Glucose (GOD-PAP method - Roche/Hitachi 912/917/ Modular: ACN 525), total cholesterol (CHOD-PAP method - Hitachi Modular: P/D: ACN 433), and triglycerides (GPO-PAP method - Hitachi 912/917/ Modular: ACN:781).

\section{Statistical analyses}

All statistical analyses were performed in the SPSS software v.18 (SPSS, Chicago, IL). Data are presented as mean \pm standard error of the mean in figures. One-way analysis of variance (ANOVA) was used for comparison of between-group differences, followed by Tukey's b test when necessary. Generalized estimating equations, with Bonferroni's post-hoc test, were used to analyze the effects of time, the differences between groups and the interaction between groups over time. Significance of a finding was assumed if $\mathrm{P}<0.05$.

\section{RESULTS}

The body weight showed no difference between the hypoxia and control experimental groups at day 1 ( $\mathrm{P}$ $=0.13$; Figure 1$)$. At day 21 , before injection protocol started, the average body weight of two groups under intermittent hypoxia, vehicle and melatonin, was significantly reduced $(\mathrm{P}<0.05)$, as opposed to the three control groups, which had experienced significant increases in weight $(\mathrm{P}=0.05)$. At day 35 , after 14 days of injections according to the protocol, animals in both the hypoxia and control groups had lost weight $(\mathrm{P}<$ $0.05)$. 


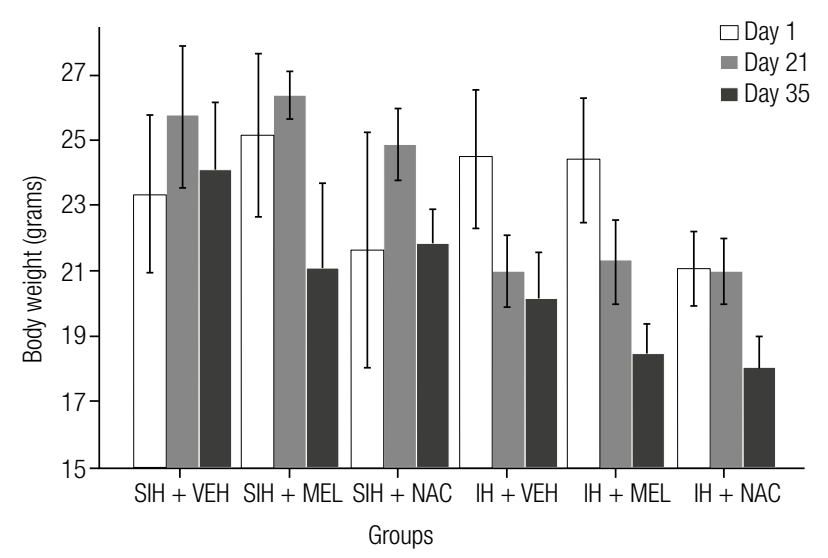

Figure 1. Body weight of mice in each group during the 35-day experimental period, shown as mean \pm one standard error of the mean. The groups under intermittent hypoxia, vehicle and melatonin exhibited weight loss in the first 21 days, while that receiving intraperitoneal injections of melatonin or $\mathrm{N}$-acetylcysteine weight loss in the next 14 days. The groups under sham hypoxia exhibited weight gain in the first 21 days and weight loss in the next 14 days, while receiving intraperitoneal injections. SIH, sham intermittent hypoxia. VEH: vehicle; MEL: melatonin; NAC: N-acetylcysteine; $\mathbb{H}$ : intermittent hypoxia.
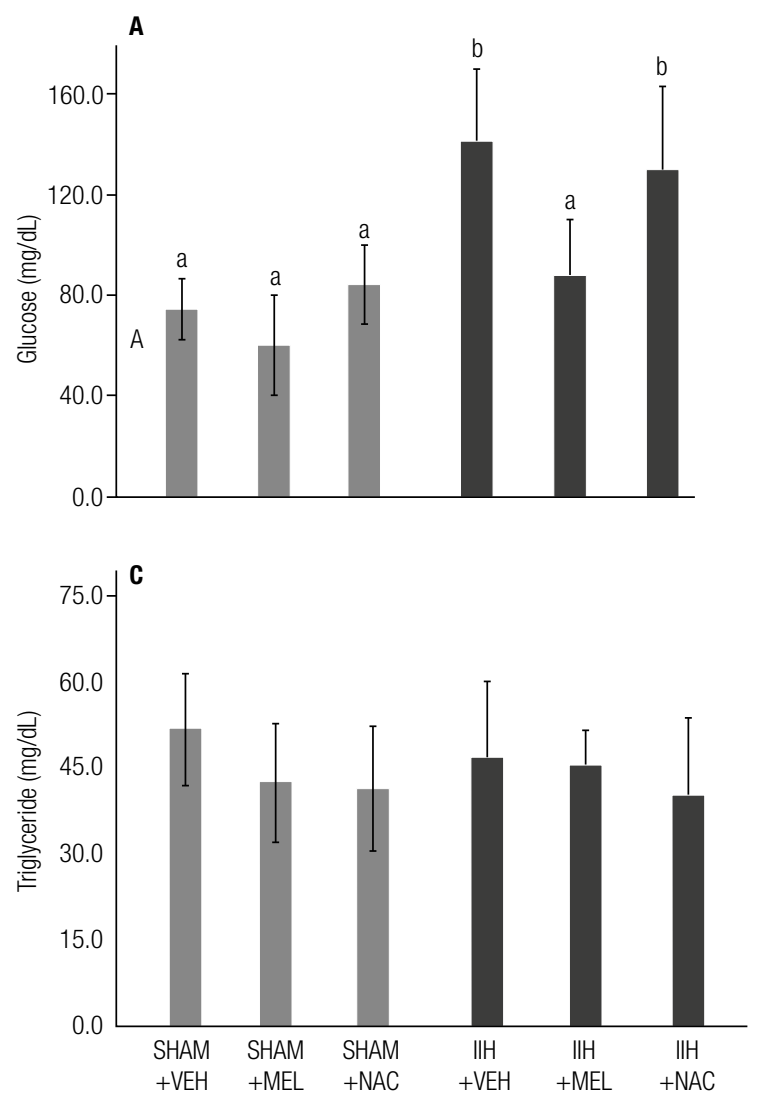

At day 35, glucose levels were significantly different between the hypoxia and control groups $(\mathrm{P}<$ 0.05 ), being highest in the hypoxia plus vehicle and hypoxia plus $\mathrm{N}$-acetylcysteine groups as compared with the remaining groups $(\mathrm{P}<0.05$; Figure $2 \mathrm{~A})$. The average glucose level in the sham hypoxia plus melatonin group was significantly lower than in the remaining control groups combined $(60 \pm 26$ vs. 79 $\pm 19 \mathrm{mg} / \mathrm{dL} ; \mathrm{P}=0.05)$. No significant differences were seen among the six groups in terms of lipid levels (Figure 2B, 2C, 2D).

\section{DISCUSSION}

The finding of fasting hyperglycemia occurring in association with intermittent hypoxia is consistent with previous reports $(5,6)$. To the best of our knowledge, this is the first report of melatonin having a role in preventing intermittent hypoxia-related hyperglycemia.
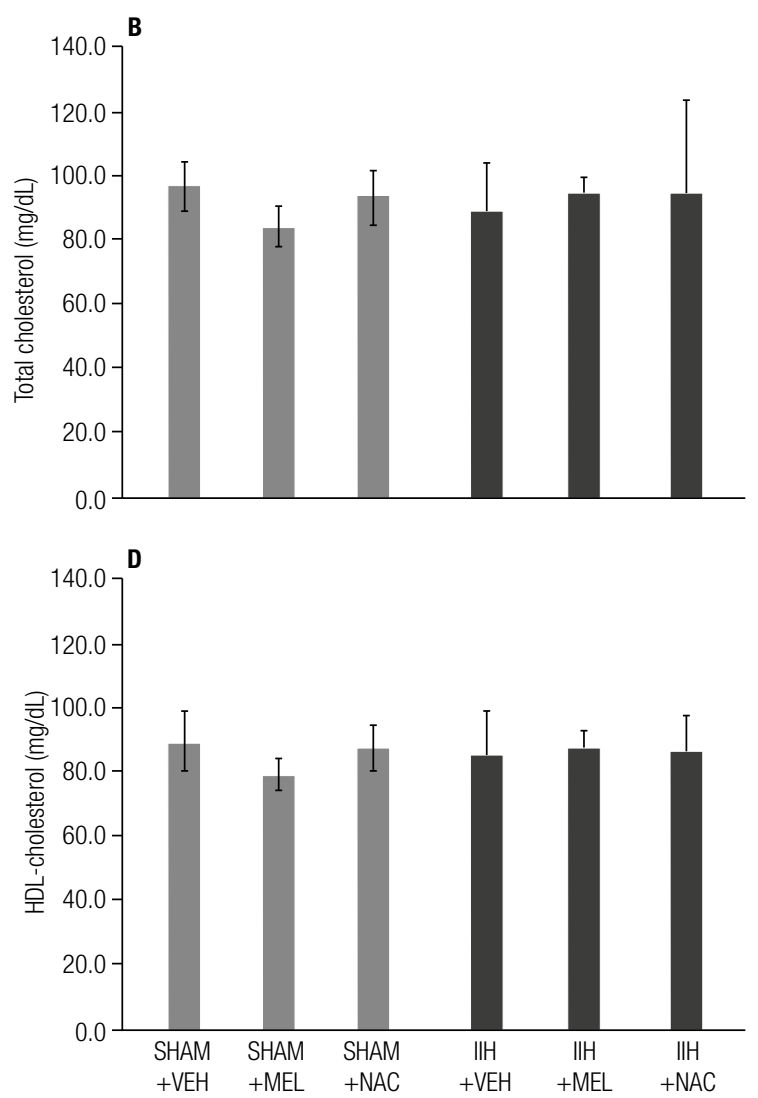

Figure 2. Biochemical parameters, expressed as mean \pm one standard error of the mean, and statistical significance of differences among the six experimental groups as quantified by one-way ANOVA. (A) blood glucose; (B) serum total cholesterol; (C) serum triglycerides; (D) serum HDL-cholesterol. Different letters on top of the bars denote differences between the groups as evaluated by Tukey's b test $(P<0.01)$. SIH: sham intermittent hypoxia; VEH: vehicle; MEL: melatonin; NAC: N-acetylcysteine; IH: intermittent hypoxia; HDL: high-density lipoprotein; IH: intermittent hypoxia. Intermittent hypoxia black bars and sham gray bars. 
The protection conferred by melatonin against intermittent-hypoxia-triggered hyperglycemia in the present study may be related either to its antioxidant role (23) or to the influence of melatonin on the control of carbohydrate metabolism $(13,14,24)$. The possibility, however, that the antioxidant properties of melatonin are at play is overshadowed by the fact that the other antioxidant, $\mathrm{N}$-acetylcysteine, had no effect on glucose levels or body weight. Furthermore, the glucose level in the control plus melatonin group was lower than in the other control groups. The most likely mechanism for the finding of normal glucose levels in the hypoxia plus melatonin group is, therefore, melatonin-mediated improvement of glucose homeostasis (25-27).

Rats (14) and mice (15) lose weight under intermittent hypoxia. The data reported herein reproduce previous findings in that a significant reduction in body weight was observed after 21 days of exposure to intermittent hypoxia. In these previous reports, the reduction in brown adipose tissue and UCP-1 mRNA expression may suggest that the weight loss may be due to impairment of energy-saving mechanisms mediated by brown adipose tissue (28). The weight loss induced by the injections from day 21 to day 35 was probably due to manipulation stress, since even the control group, receiving vehicle injections, lost weight._

The discovery of an effect of melatonin on hypoxia-induced hyperglycemia serves as a basis for future mechanistic studies. The present study, however, has limitations. The elevated glucose levels in the hypoxia plus vehicle and hypoxia plus $\mathrm{N}$-acetyscisteine groups were sustained after 8 hours or more of fasting. Although prolonged fasting is almost certainly stressful for the mice, the metabolic overload sustained by the animals was similar in all groups. Additionally, longer fasting time would allow a longer time for the glucose level to lower. Therefore, this systematic cause of error would be conservative, tending to eliminate the differences.

Also, our study was designed to explore glucose and lipid levels, but no changes were seen in the lipid profile. The induction of hyperlipidemia by intermittent hypoxia occurs under severe hypoxia ( $5 \%$ nadir of inspired $\mathrm{O}_{2}$ ) but not under moderate hypoxia (10\%) (29). The finding of similar cholesterol and triglyceride levels in all groups in the present study can be explained by the nadir of inspired $\mathrm{O}_{2}$ of $7 \%$ selected for this study being insufficient to induce hyperlipidemia. The initial objective of assessing lipid levels consumed most of the small volume of blood withdrawn from each mouse, preventing investigation of oxidative stress and insulin resistance. The absence of an effect of $\mathrm{N}$-acetylcysteine limited interpretation of the effects of melatonin on glycemia. Further research is necessary to elucidate the mechanisms whereby hyperglycemia prevention was elicited only by melatonin. To explore the complex system of glycemic control, one must gauge, at the very least, insulin, norepinephrine, and glucagon levels; if available, these measurements would have furthered our knowledge of the mechanisms underlying the hyperglycemia observed in the present study. Since lipids were not affected in this model, future efforts should focus on explaining the effects of melatonin on glycemic control under intermittent hypoxia.

In summary, these findings suggest that exposure to intermittent hypoxia, simulating sleep apnea, for 35 days promotes high plasma glucose levels, which were not prevented by $\mathrm{N}$-acetylcysteine. In mice receiving melatonin, normal glucose levels were observed under intermittent hypoxia. Whether the effect of melatonin is due to a direct action on glycemic control or to influences on oxidative stress has yet to be elucidated.

Author contributions: Renata Schenkel Rivera Kaminski, Denis Martinez, Cintia Zappe Fiori, Darlan Pase Rosa, Norma Possa Marroni contributed to the design and performance of the experiment, the analysis of data, and preparation of the manuscript. Emerson Ferreira Martins, Micheli Fagundes, Carolina Caruccio Montanari contributed to the analysis of data and to the manuscript preparation.

Acknowledgments: this work was supported by the Research Incentive Fund of the Hospital de Clínicas de Porto Alegre.

Disclosure: no potential conflict of interest relevant to this article was reported.

\section{REFERENCES}

1. Dempsey JA, Veasey SC, Morgan BJ, O'Donnell CP. Pathophysiology of sleep apnea. Physiol Rev. 2010;90(1):47-112.

2. Shaw JE, Punjabi NM, Wilding JP, Alberti KG, Zimmet PZ; International Diabetes Federation Taskforce on Epidemiology and Prevention. Sleep-disordered breathing and type 2 diabetes: a report from the International Diabetes Federation Taskforce on Epidemiology and Prevention. Diabetes Res Clin Pract. 2008;81(1):2-12.

3. Lecomte $P$, Criniere L, Fagot-Campagna A, Druet C, Fuhrman C. Underdiagnosis of obstructive sleep apnoea syndrome in patients with type 2 diabetes in France: ENTRED 2007. Diabetes Metab. 2013;39(2):139-47.

4. Aronsohn RS, Whitmore H, Van Cauter E, Tasali E. Impact of untreated obstructive sleep apnea on glucose control in type 2 diabetes. Am J Respir Crit Care Med. 2010;181(5):507-13.

5. Xu W, Chi L, Row BW, Xu R, KeY, Xu B, et al. Increased oxidative stress is associated with chronic intermittent hypoxia-mediated 
brain cortical neuronal cell apoptosis in a mouse model of sleep apnea. Neuroscience. 2004;126(2):313-23.

6. Drager LF, Jun JC, Polotsky VY. Metabolic consequences of intermittent hypoxia: relevance to obstructive sleep apnea. Best Pract Res Clin Endocrinol Metab. 2010;24(5):843-51.

7. Pallayova M, Banerjee D, Taheri S. Novel insights into metabolic sequelae of obstructive sleep apnoea: a link between hypoxic stress and chronic diabetes complications. Diabetes Res Clin Pract. 2014;104(2):197-205.

8. Kendzerska T, Gershon AS, Hawker G, Tomlinson G, Leung RS. Obstructive sleep apnea and incident diabetes. A historical cohort study. Am J Respir Crit Care Med. 2014 Jul 15;190(2):218-25.

9. Louis M, Punjabi NM. Effects of acute intermittent hypoxia on glucose metabolism in awake healthy volunteers. J Appl Physiol (1985). 2009;106(5):1538-44.

10. liyori N, Alonso LC, Li J, Sanders MH, Garcia-Ocana A, O'Doherty $\mathrm{RM}$, et al. Intermittent hypoxia causes insulin resistance in lean mice independent of autonomic activity. Am J Respir Crit Care Med. 2007;175(8):851-7.

11. Drager LF, Li J, Reinke C, Bevans-Fonti S, Jun JC, Polotsky VY. Intermittent hypoxia exacerbates metabolic effects of diet-induced obesity. Obesity (Silver Spring). 2011;19(11):2167-74.

12. Bertuglia $\mathrm{S}$, Reiter RJ. Melatonin reduces microvascular damage and insulin resistance in hamsters due to chronic intermittent hypoxia. J Pineal Res. 2009;46(3):307-13.

13. Winiarska K, Fraczyk T, Malinska D, Drozak J, Bryla J. Melatonin attenuates diabetes-induced oxidative stress in rabbits. J Pineal Res. 2006;40(2):168-76.

14. Peschke $E$, Mühlbauer $E$. New evidence for a role of melatonin in glucose regulation. Best Pract Res Clin Endocrinol Metab. 2010;24(5):829-41.

15. Aruoma OI, Halliwell B, Hoey BM, Butler J. The antioxidant action of $\mathrm{N}$-acetylcysteine: its reaction with hydrogen peroxide, hydroxyl radical, superoxide, and hypochlorous acid. Free Radic Biol Med. 1989;6(6):593-7.

16. Kamboj SS, Vasishta RK, Sandhir R. N-acetylcysteine inhibits hyperglycemia-induced oxidative stress and apoptosis markers in diabetic neuropathy. J Neurochem. 2010;112(1):77-91.

17. Wang T, Qiao S, Lei S, Liu Y, Ng KF, Xu A, et al. N-acetylcysteine and allopurinol synergistically enhance cardiac adiponectin content and reduce myocardial reperfusion injury in diabetic rats. PLoS One. 2011;6(8):e23967.

18. Baronio D, Martinez D, Fiori CZ, Bambini-Junior V, Forgiarini LF, Pase da Rosa D, et al. Altered aquaporins in the brains of mice submitted to intermittent hypoxia model of sleep apnea. Respir Physiol Neurobiol. 2013;15;185(2):217-21.

19. Martinez D, Fiori CZ, Baronio D, Carissimi A, Kaminski RS, Kim LJ, et al. Brown adipose tissue: is it affected by intermittent hypoxia? Lipids Health Dis. 2010;19;(9):121.

20. Hawkins P, Morton DB, Burman O, Dennison N, Honess P, Jennings $M$, et al. UK Joint Working Group on Refinement BVAAWF/ FRAME/RSPCA/UFAW. A guide to defining and implementing protocols for the welfare assessment of laboratory animals: eleventh report of the BVAAWF/FRAME/RSPCA/UFAW Joint Working Group on Refinement. Lab Anim. 2011;45(1):1-13.

21. Guven A, Yavuz O, Cam M, Ercan F, Bukan N, Comunoglu C, et al. Effects of melatonin on streptozotocin-induced diabetic liver injury in rats. Acta Histochem. 2006;108(2):85-93.

22. AVMA releases updated euthanasia guidelines. J Am Vet Med Assoc. $2007 ; 231: 827$.

23. Tan DX, Manchester LC, Terron MP, Flores LJ, Reiter RJ. One molecule, many derivatives: a never-ending interaction of melatonin with reactive oxygen and nitrogen species? J Pineal Res. 2007;42(1):28-42.

24. Muhlbauer E, Gross E, LabucaYK, Wolgast S, Peschke E. Loss of melatonin signalling and its impact on circadian rhythms in mouse organs regulating blood glucose. Eur J Pharmacol. 2009;606(1-3):61-71.

25. Sartori C, Dessen P, Mathieu C, Monney A, Bloch J, Nicod P, et al. Melatonin improves glucose homeostasis and endothelial vascular function in high-fat diet-fed insulin-resistant mice. Endocrinology. 2009;150(12):5311-7.

26. Bizot-Espiard JG, Doublé A, Guardiola-Lemaitre B, Delagrange $P$, Ktorza A, Pénicaud L. Diurnal rhythms in plasma glucose, insulin, growth hormone and melatonin levels in fasted and hyperglycaemic rats. Diabetes Metab. 1998;24(3):235-40.

27. Koziróg M, Poliwczak AR, Duchnowicz P, Koter-Michalak M, Sikora $\mathrm{J}$, Broncel M. Melatonin treatment improves blood pressure, lipid profile, and parameters of oxidative stress in patients with metabolic syndrome. J Pineal Res. 2011;50(3):261-6.

28. Fiori CZ, Martinez D, Baronio D, da Rosa DP, Kretzmann NA, Forgiarini LF, et al. Downregulation of uncoupling protein-1 mRNA expression and hypoadiponectinemia in a mouse model of sleep apnea. Sleep Breath. 2014;18(3):541-8.

29. Li J, Savransky V, Nanayakkara A, Smith PL, O"Donnell CP, Polotsky VY. Hyperlipidemia and lipid peroxidation are dependent on the severity of chronic intermittent hypoxia. J Appl Physiol. 2007;102(2):557-63. 